\title{
Early stages of the metal-to-insulator transition of a thin $\mathrm{V}_{2} \mathrm{O}_{3}$ film
}

Cite as: J. Appl. Phys. 103, 063705 (2008); https://doi.org/10.1063/1.2871302

Submitted: 26 December 2006 • Accepted: 19 December 2007 • Published Online: 19 March 2008

C. Müller, A. A. Nateprov, M. Klemm, et al.

\section{ARTICLES YOU MAY BE INTERESTED IN}

Direct observation of the lattice precursor of the metal-to-insulator transition in $\mathrm{V}_{2} \mathrm{O}_{3}$ thin

films by surface acoustic waves

Applied Physics Letters 102, 101904 (2013); https://doi.org/10.1063/1.4794948

Surface acoustic wave investigations of the metal-to-insulator transition of $\mathrm{V}_{2} \mathrm{O}_{3}$ thin films on lithium niobate

Journal of Applied Physics 98, 084111 (2005); https://doi.org/10.1063/1.2103410

Tuning metal-insulator transitions in epitaxial $\mathrm{V}_{2} \mathrm{O}_{3}$ thin films

Applied Physics Letters 112, 161902 (2018); https://doi.org/10.1063/1.5023180

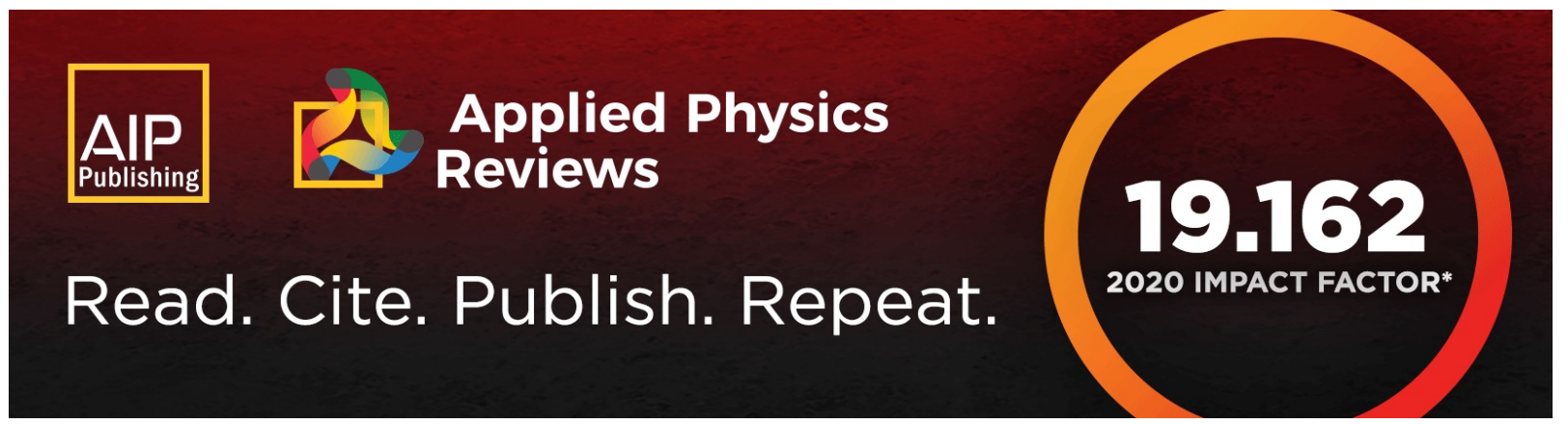

J. Appl. Phys. 103, 063705 (2008); https://doi.org/10.1063/1.2871302

103, 063705

(C) 2008 American Institute of Physics. 


\title{
Early stages of the metal-to-insulator transition of a thin $\mathbf{V}_{\mathbf{2}} \mathrm{O}_{\mathbf{3}}$ film
}

\author{
C. Müller, ${ }^{\text {a) }}$ A. A. Nateprov, M. Klemm, A. Wixforth, R. Tidecks, and S. Horn \\ Institut für Physik, Universität Augsburg, Universitätsstrasse 1, D-86159 Augsburg, Germany
}

(Received 26 December 2006; accepted 19 December 2007; published online 19 March 2008)

\begin{abstract}
The metal-to-insulator (MI) transition of a $\mathrm{V}_{2} \mathrm{O}_{3}$ thin film is studied, using a surface acoustic wave delay line. The $\mathrm{V}_{2} \mathrm{O}_{3}$ film covers not only the sound path but also one of the interdigital transducers (IDTs). The resulting mismatch of the IDTs detunes the delay line, until the film passes through the MI transition. This "self-retuning" behavior makes the device extremely sensitive to the initial changes of the electrical and dielectric properties of the film during the MI transition. Attenuation and sound velocity are measured between 260 and $4.2 \mathrm{~K}$. Both suggest a precursor to the MI transition in the paramagnetic metallic regime. The existence of a precursor is consistent with recent extended x-ray absorption fine structure measurements. An oscillatory behavior with steep reductions of the sound velocity is observed during the MI transition. The reductions of the sound velocity probably indicate the recently predicted anomaly at the Mott transition. (C) 2008 American Institute of Physics. [DOI: 10.1063/1.2871302]
\end{abstract}

\section{INTRODUCTION}

Although widely investigated, the metal-to-insulator (MI) transition of $\mathrm{V}_{2} \mathrm{O}_{3}$ is not completely understood. ${ }^{1-12}$ An interpretation in terms of a typical Mott-Hubbard system $^{13-16}$ appears questionable because structural, electronic, and magnetic degrees of freedom are involved. ${ }^{17}$

The volume increases in the percentage ${ }^{18}$ range at the MI transition, resulting in cracks or even destruction of single crystals so that ultrasound experiments are almost nonexistent. The structural integrity of thin films, however, is hardly affected. Therefore, we recently deposited $\mathrm{V}_{2} \mathrm{O}_{3}$ thin films on a piezoelectric substrate. This allows the investigation using surface acoustic waves (SAWs). ${ }^{19}$

In the present work, we focus on a detailed study of a lattice precursor to the MI transition, using a self-retuning SAW delay line. This precursor in the paramagnetic metallic regime was found in Ref. 19 and in extended x-ray absorption fine structure measurements ${ }^{20,21}$ and has meanwhile been confirmed by Brillouin scattering. ${ }^{22}$

In the context of the present studies, evidence was found for the sound velocity anomaly recently predicted by the compressible Hubbard model. ${ }^{23}$

\section{SAMPLE PREPARATION AND MEASURING TECHNIQUES}

\section{A. Thin film preparation and experimental setup}

The $\mathrm{V}_{2} \mathrm{O}_{3}$ film (thickness $d=300 \mathrm{~nm}$ ) was deposited on a $128^{\circ}$ rotated $Y X$ cut $\mathrm{LiNbO}_{3}$ substrate. As in Ref. 19, x-ray diffraction measurements indicate a reduced $c$ parameter (describing the structure of $\mathrm{V}_{2} \mathrm{O}_{3}$ as a distorted hexagonal lattice $^{24}$ ) due to stress.

The SAW used to investigate the properties of the $\mathrm{V}_{2} \mathrm{O}_{3}$ film was electrically excited and detected by two identical

\footnotetext{
a) Author to whom correspondence should be addressed. Electronic mail: claus.mueller@physik.uni-augsburg.de. Tel.: +49(0)821/598-2111. FAX: +49(0)821/598-3411.
}

interdigital transducers $^{25}$ (IDTs) with split-four-finger electrodes. The SAW delay line geometry is sketched in Fig. 1. In addition to the sound path, one IDT was covered by the $\mathrm{V}_{2} \mathrm{O}_{3}$ film. The end of the finger electrode of the covered IDT is shown in Fig. 2.

Although one IDT is covered by $\mathrm{V}_{2} \mathrm{O}_{3}$, there is an electrical high frequency impedance matching for both IDTs, allowing for the reflection-free electrical excitation of the SAW by applying a radio frequency voltage. The electrical impedance is $\sim 50 \Omega$ at room temperature at the fundamental frequency (see Sec. II B) for the covered as well as for the uncovered IDT. The reason probably is that the covered IDT is not completely short circuited by the $\mathrm{V}_{2} \mathrm{O}_{3}$ film and that
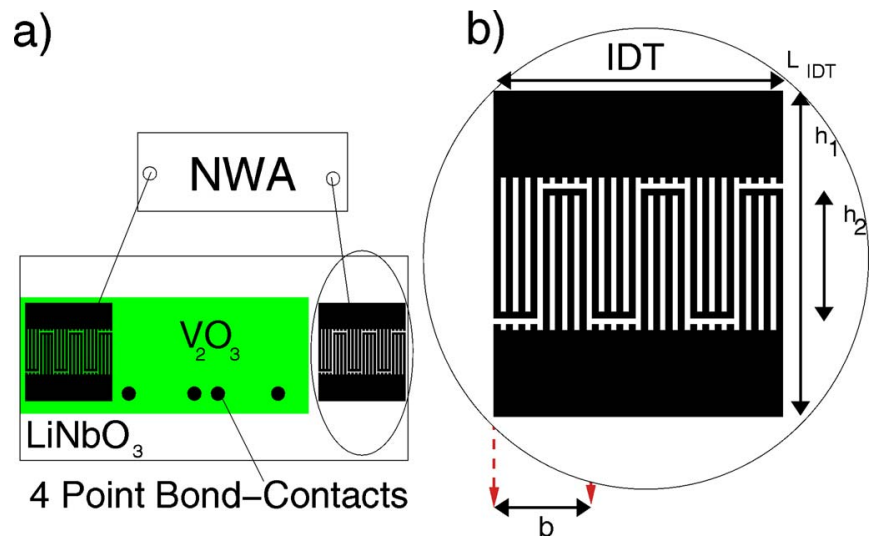

FIG. 1. (Color online) (a) Sketch of a SAW transmission line with a $\mathrm{V}_{2} \mathrm{O}_{3}$ film on top of $\mathrm{LiNbO}_{3}$; (b) enlarged view of the IDT structure. The left IDT is covered by the $\mathrm{V}_{2} \mathrm{O}_{3}$ film. Length of the film $L_{F \text {,tot }}=5.86 \mathrm{~mm}$, width $w$ $=3.6 \mathrm{~mm}$, and IDTs centered at $\sim w / 2$ of the film. Length of the sound path between the IDTs covered by the $\mathrm{V}_{2} \mathrm{O}_{3}$ film: $L_{F}=4.86 \mathrm{~mm}$. Bond contacts (Al wire, $50 \mu \mathrm{m}$ diameter) serve for dc resistance measurements [current leads at $\sim 1.54$ and $0.99 \mathrm{~mm}$ from left and right corners of the $\mathrm{V}_{2} \mathrm{O}_{3}$ film, respectively; voltage probes with distance $320 \mu \mathrm{m}$ (midpoint to midpoint) approximately in the middle between the current leads]. Dimensions of the IDTs: $h_{1} \sim 1.14 \mathrm{~mm}, h_{2} \sim 0.6 \mathrm{~mm}, L_{\mathrm{IDT}} \sim 0.4 \mathrm{~mm}$ (space between right IDT and film $\sim 0.18 \mathrm{~mm}$; distance left border of film to right side of left IDT $\sim 0.82 \mathrm{~mm}$ ). NWA: vector network analyzer (type ZVC, Rohde and Schwarz). 


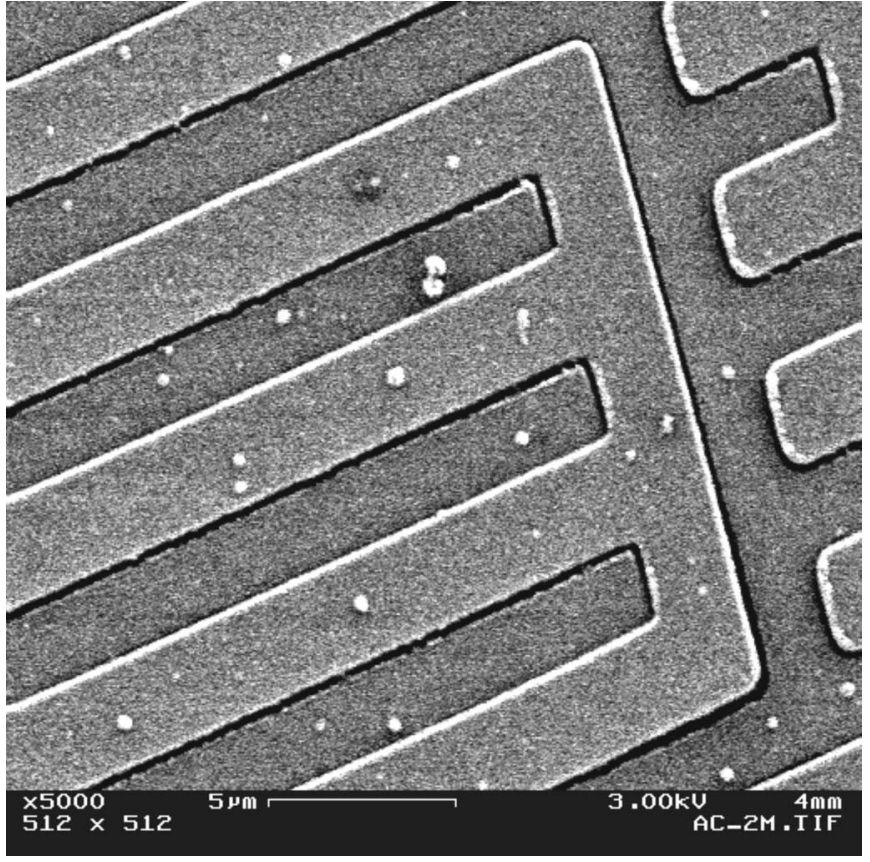

FIG. 2. Scanning electron microscope image of a $\mathrm{V}_{2} \mathrm{O}_{3}$ thin film deposited on $\mathrm{LiNbO}_{3}$. Finger region of the covered IDT (picture width: $\sim 23 \mu \mathrm{m}$ ).

the resistivity of the $\mathrm{V}_{2} \mathrm{O}_{3}$ film at room temperature is about 1000 times larger than that of the aluminum fingers of the IDT. The incomplete short circuiting is presumably caused by the fine slits between finger electrodes and film (see Fig. 2 ). This may be also the reason for the high dc resistance of around $300 \Omega$ measured across the electrodes of the covered IDT.

The measuring technique is basically described in Ref. 19. The attenuation of the SAW at the frequency $f_{t}$, which has the highest transmission intensity, was read out of a vector network analyzer (NWA) [Fig. 1(a))]. The group velocity was obtained in the same run by a second channel of the NWA. The resistance $R$ of the film was measured by a standard four point dc method. Investigations between 260 and $4.2 \mathrm{~K}$ were performed in a cryostat with a variable temperature inset.

\section{B. Self-retuning of the SAW delay line}

The best transmitted frequency $f_{t}$ (frequency for minimum attenuation of the SAW) is determined by the transfer functions of both IDTs. Since one IDT is covered with $\mathrm{V}_{2} \mathrm{O}_{3}$, it is not simply the best emitted ("fundamental") frequency $f_{0}$, given by the relation $\lambda f_{0}=v$, with $v$ equal to the sound velocity $v_{1}$ of $\mathrm{LiNbO}_{3}$ covered by the metallic structure of the IDT or to the velocity $v_{2}$ of $\mathrm{LiNbO}_{3}$ covered with the IDT structure and with $\mathrm{V}_{2} \mathrm{O}_{3}$. Here, $\lambda=34.64 \mu \mathrm{m}$ [equal to the spacing $b$ of the finger electrodes in Fig. 1(b)] is the wavelength of the SAW emitted by the IDT. The velocities $v_{1}$ and $v_{2}$ are in the range between $v_{0}=3978.2 \mathrm{~m} / \mathrm{s}$ for $\mathrm{LiNbO}_{3}$ and $v_{\mathrm{sc}}=3870 \mathrm{~m} / \mathrm{s}$ for short circuited $\mathrm{LiNbO}_{3}$ at room temperature. ${ }^{26}$

Therefore, there is a frequency mismatch between the IDTs caused by the metallic conductivity of the $\mathrm{V}_{2} \mathrm{O}_{3}$ film covering one of them. Moreover, the degree of metallization

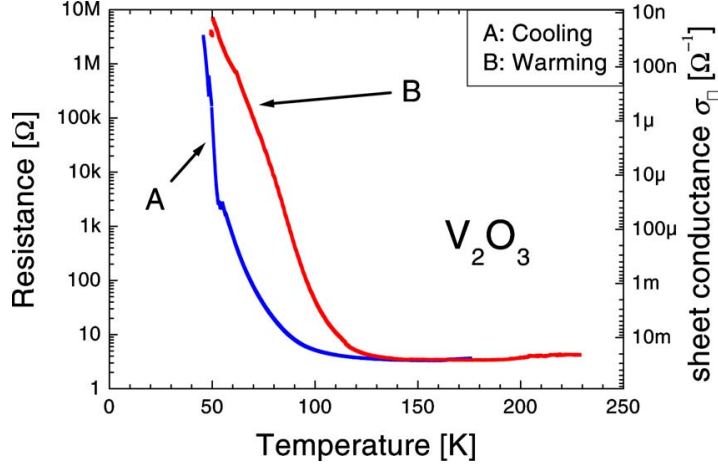

FIG. 3. (Color online) Temperature dependence of the resistance (coolingwarming cycle) and sheet conductance $\sigma_{\square}$, respectively, of a $\mathrm{V}_{2} \mathrm{O}_{3}$ film on $\mathrm{LiNbO}_{3}$ substrate.

influences the emission and detection characteristics of the IDT, as discussed in detail in Ref. 27 (Chap. 4.2.2) and Ref. 28. Thus, the delay line is detuned until it retunes when the $\mathrm{V}_{2} \mathrm{O}_{3}$ film passes through the MI transition. Due to this "selfretuning" behavior coupled to the MI transition, the delay line is extremely sensitive to small initial changes of electrical and dielectric properties of the film during the MI transition.

\section{RESULTS AND DISCUSSION}

\section{A. Resistance}

The measured dc resistance $R$ and the corresponding sheet conductance $\sigma_{\square}$ (calculated as described in Ref. 19) of the $\mathrm{V}_{2} \mathrm{O}_{3}$ film are shown in Fig. 3. Due to stress, the transition temperature $T_{\mathrm{MI}}$ is lower than that for the stoichometric bulk material of about $170 \mathrm{~K}$ for cooling. ${ }^{9,12}$ The hysteresis (cooling/warming) has about twice the width compared to that of a single crystal. ${ }^{29}$

\section{B. Attenuation}

In the same run with the dc resistance, the attenuation of the SAW was measured at the best transmitted frequency $f_{t}$. Since $f_{t}$ is adjusted to the change of the attenuation of the SAW caused by the varying properties of the $\mathrm{V}_{2} \mathrm{O}_{3}$ film, this frequency varies with temperature. The range extends from 114.6 $\mathrm{MHz}$ at $4.2 \mathrm{~K}$ (where $\mathrm{V}_{2} \mathrm{O}_{3}$ behaves like a dielectric) to $112.9 \mathrm{MHz}$ at $250 \mathrm{~K}$ (where $\mathrm{V}_{2} \mathrm{O}_{3}$ had become metallic). A strong change of $f_{t}$ is observed at $T_{\mathrm{MI}}$ (as expected $\left.{ }^{30}\right)$.

The result of the attenuation measurement is plotted in Fig. 4(a). The attenuation shows a thermal hysteresis (as the dc resistance during the MI transition) with a maximum at $55.5 \mathrm{~K}$ on cooling and at $81 \mathrm{~K}$ on warming. In addition, there are minima at $67 \mathrm{~K}$ for cooling and $94 \mathrm{~K}$ for warming.

For comparison, the theoretical curves (cooling and warming) are displayed in Fig. 4(b). These curves were calculated according to Ref. 19 using the absorption coefficient, $\Gamma=K^{2}(\pi / \lambda)\left(\sigma_{\square} / \sigma_{m}\right)\left[1+\left(\sigma_{\square} / \sigma_{m}\right)^{2}\right]^{-1}$, obtained from the theory of Ingebrigtsen. ${ }^{19,31}$ Here, $K^{2}=0.056$ is the electromechanical coupling constant (Ref. 27, Table 3.2). The sheet conductance has been taken from the dc resistance measurement (Fig. 3). The attenuation limit measured at the lowest temperature [see Fig. 4(a)] in the insulating state of the $\mathrm{V}_{2} \mathrm{O}_{3}$ 

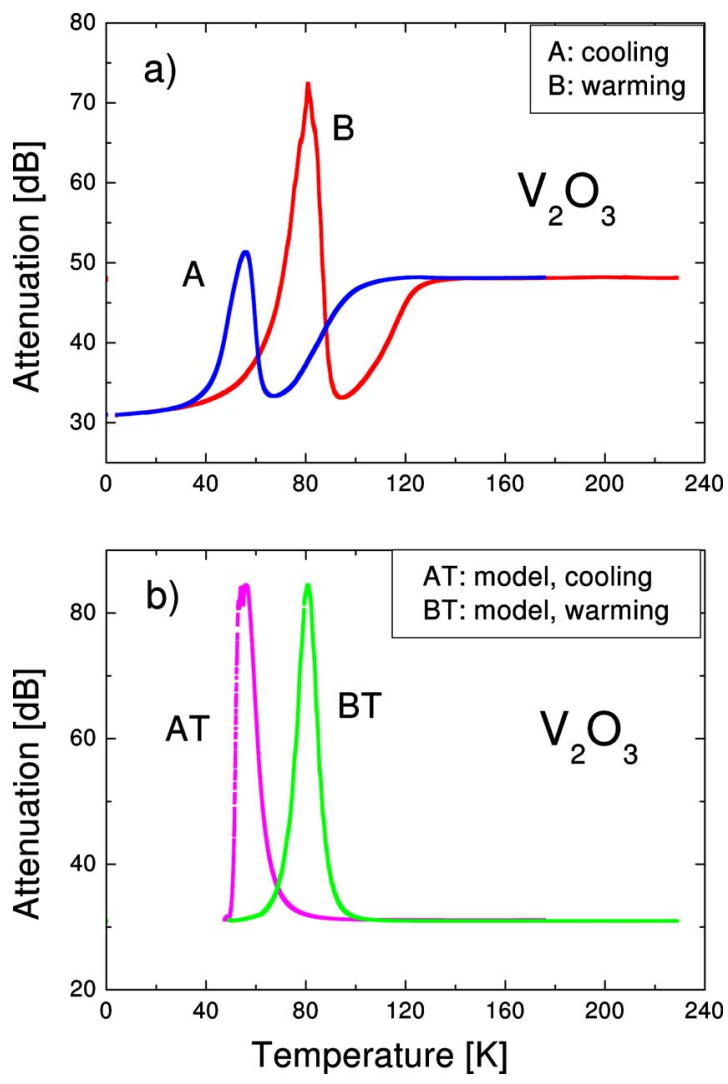

FIG. 4. (Color online) Comparison of (a) experimental and (b) calculated values for the attenuation of the SAW. The onset of the MI transition is clearly visible.

film has been added as an offset (insertion loss). In the present work (like in Ref. 19), we always plot and discuss the absolute value of the attenuation.

The absorption coefficient, and thus the attenuation, becomes maximal at $\sigma_{\square}=\sigma_{m} \cdot{ }^{32}$ Calculated and measured positions of the maximum of the attenuation match, if we use $\sigma_{m}=4.5 \times 10^{-5}$ and $1.3 \times 10^{-5} \Omega^{-1}$, as observed for the sheet resistance at 55.5 and $81 \mathrm{~K}$ in Fig. 3 for cooling and warming, respectively.

The values of $\sigma_{m}$ for cooling and warming differ from each other, and they are one order of magnitude higher than $\sigma_{m}=2 \times 10^{-6} \Omega^{-1}$ expected for the piezoelectric $\mathrm{LiNbO}_{3}$ substrate used. The reason may be that the dc measurements yield a sheet conductance deviating from the average value (sensed by the SAW), due to percolation effects, with different paths for increasing and decreasing temperature. Moreover, the value of $\sigma_{m}$ (which depends on $v_{0}$ and the dielectric constants of the substrate ${ }^{19}$ ) may be changed in the presence of the $\mathrm{V}_{2} \mathrm{O}_{3}$ film. Finally, the relative permittivity of the $\mathrm{V}_{2} \mathrm{O}_{3}$ film, which is neglected in the limit of the theory used here, may be of importance. This is possibly also the reason why the height of the calculated attenuation maxima is equal for increasing and decreasing temperatures, contrary to the experimental observation.

The measured attenuation well above the MI transition is constant in a wide temperature range, with a value much higher than the insertion loss mentioned above. Then, a pronounced minimum is observed just above the MI transition for cooling and warming. This behavior is not displayed in

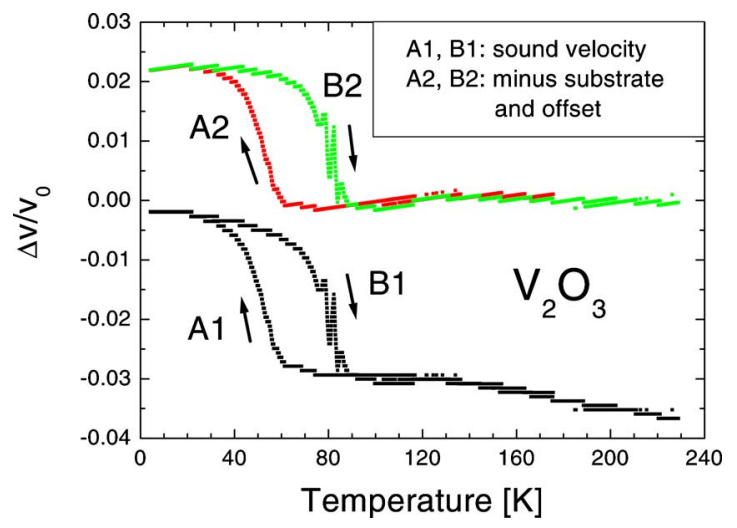

FIG. 5. (Color online) Velocity shift vs temperature for cooling and warming: as measured (A1, B1) and minus substrate in linear order and offset (A2, B2).

the curves calculated from the theory. It originates from changes of the electrical and dielectric properties caused by the MI transition of the $\mathrm{V}_{2} \mathrm{O}_{3}$ layer deposited directly onto one of the IDTs. These changes are related to the very beginning, i.e., the early stages of the MI transition, giving rise to a readjustment of the best emitted frequency of the covered IDT to that one of the uncovered IDT. As we discussed in Sec. II B, this retuning of the SAW delay line by the MI transition itself is a highly sensitive method to detect even small changes in the $\mathrm{V}_{2} \mathrm{O}_{3}$ film.

The temperature range in which the decrease of the measured attenuation and the formation of a minimum is observed corresponds to the range of an anomaly detected in the sound velocity of the SAW. This will be discussed in the next sections.

\section{Sound velocity: Lattice precursor to the MI transition}

In Fig. 5 (curves A1 and B1), we plotted the normalized sound velocity shift $\Delta v / v_{0}$, where $\Delta v=v-v_{0}$, caused by the $\mathrm{V}_{2} \mathrm{O}_{3}$ thin film, compared to the case of a freely propagating SAW on the surface of an uncovered $\mathrm{LiNbO}_{3}$ substrate. Here, $v$ is the velocity in the region of the sound path covered by the $\mathrm{V}_{2} \mathrm{O}_{3}$ thin film, obtained by dividing the length of the covered region by the corresponding traveling time of the SAW.

To obtain curves A2 and B2 in Fig. 5, the slightly temperature dependent sound velocity shift for an uncovered $\mathrm{LiNbO}_{3}$ delay line ${ }^{33}$ was subtracted from the data of A1 and $\mathrm{B} 1$, respectively, using a linear fit to the data of the clean substrate. In addition, $\Delta v / v_{0}$ was set to zero at $250 \mathrm{~K}$ by subtracting the negative offset.

In the theory, discussed in Ref. 19, the sound velocity shift is calculated relative to the velocity $v_{\mathrm{sc}}$ of a SAW in a short circuited substrate, i.e., a nonpiezoelectric medium, which has the same properties except the piezoelectricity. To get this velocity shift from our measurement, $\Delta v=v-v_{0}$ has to be replaced $\Delta v=v-v_{\mathrm{sc}}$. However, this would only give an additional additive temperature independent term $\left(v_{0}\right.$ $\left.-v_{\mathrm{sc}}\right) / v_{0}=0.0272$, shifting curves A1 and B1 to higher val- 
ues. The reason is that $v-v_{\mathrm{sc}}=v-v_{0}+\left(v_{0}-v_{\mathrm{sc}}\right)$ with $v_{\mathrm{sc}}$ $=3870 \mathrm{~m} / \mathrm{s}^{26}$ Thus, we would get the same curves A2 and B2 as before.

The theory yields ${ }^{19} \Delta v / v_{0}=\left(K^{2} / 2\right)\left[1+\left(\sigma_{\square} / \sigma_{m}\right)^{2}\right]^{-1}$, where $\Delta v=v-v_{\mathrm{sc}}$. The maximal normalized sound velocity shift is then ${ }^{36}\left(v_{0}-v_{\mathrm{sc}}\right) / v_{0}=K^{2} / 2$. According to Ingebrigtsen $^{34}$ (see also Refs. 32 and 35), one would expect $v_{\text {sc }}$ in the denominator of the left hand side of these equations. In this case, $K^{2}$ has to be replaced by $K_{\mathrm{sc}}^{2}$, resulting from $K_{\mathrm{sc}}^{2} / 2=\left(v_{0}-v_{\mathrm{sc}}\right) / v_{\mathrm{sc}}$. However, as in the present work, usually ${ }^{27,36,37} K^{2}$ is used, requiring $v_{0}$ in the denominator.

The theoretical curve for $\Delta v / v_{0}$ is practically zero at $230 \mathrm{~K}$ and almost temperature independent for decreasing temperature, except for a steplike increase of $\left(v_{0}-v_{\mathrm{sc}}\right) / v_{0}$ $=K^{2} / 2$ at the MI transition. At around $120 \mathrm{~K}$, the experimental data in Fig. 5 curve A2 start to deviate from the theory because they go through a slight minimum. As in Ref. 19, this deviation can be fitted by a polynomial of second order. After subtracting the deviation, the jump of the experimental curve at the MI transition is nearly equal to the height predicted by the theory. For increasing temperature (Fig. 5, curve B2), a similar deviation from the theory is observed.

The minima in the sound velocity shift were also observed for both IDTs not covered by $\mathrm{V}_{2} \mathrm{O}_{3} .{ }^{19}$ The phenomenon was interpreted as a "lattice precursor" to the MI transition due to an interplay between orbital and lattice degrees of freedom. ${ }^{19}$ The reason was that a model of a piezoelectric material coated with a thin film, based exclusively on the conductivity of the $\left(\mathrm{V}_{2} \mathrm{O}_{3}\right)$ thin film (also considered in the present work), is not sufficient to explain the behavior close to the MI transition.

\section{Evidence for a lattice precursor in attenuation measurements}

Our former SAW attenuation measurements ${ }^{19}$ appeared to be not sensitive enough to show the lattice precursor of the MI transition, observed in the sound velocity investigations. The self-retuning delay line device used in the present work, however, turns out to be so sensitive that it is possible to display this phenomenon clearly in the attenuation.

In Fig. 6, the attenuation has been plotted together with the sound velocity shift. For cooling and warming, there is a coincidence of the decay of the attenuation and the sound velocity shift well above the MI transition. Thus, the attenuation reveals the MI transition precursor.

As we assume that the decay of the attenuation is caused by a change of the electrical properties of the $\mathrm{V}_{2} \mathrm{O}_{3}$ film on top of the covered IDT, the precursor should also be visible in the dc conductance measurements of our $\mathrm{V}_{2} \mathrm{O}_{3}$ film. It should appear in the temperature region around $140 \mathrm{~K}$, where the hysteretic behavior starts, i.e., the sheet conductance for cooling and warming deviate from each other [see Fig. 7(a), in which we plotted the data of Fig. 3 in a linear scale]. Above this point, the MI transition has finished for increasing temperature and has not yet started for decreasing temperature.

Below $\sim 140 \mathrm{~K}$, the sheet conductance [Fig. 7(a)] smoothly decreases with decreasing temperature and the at-
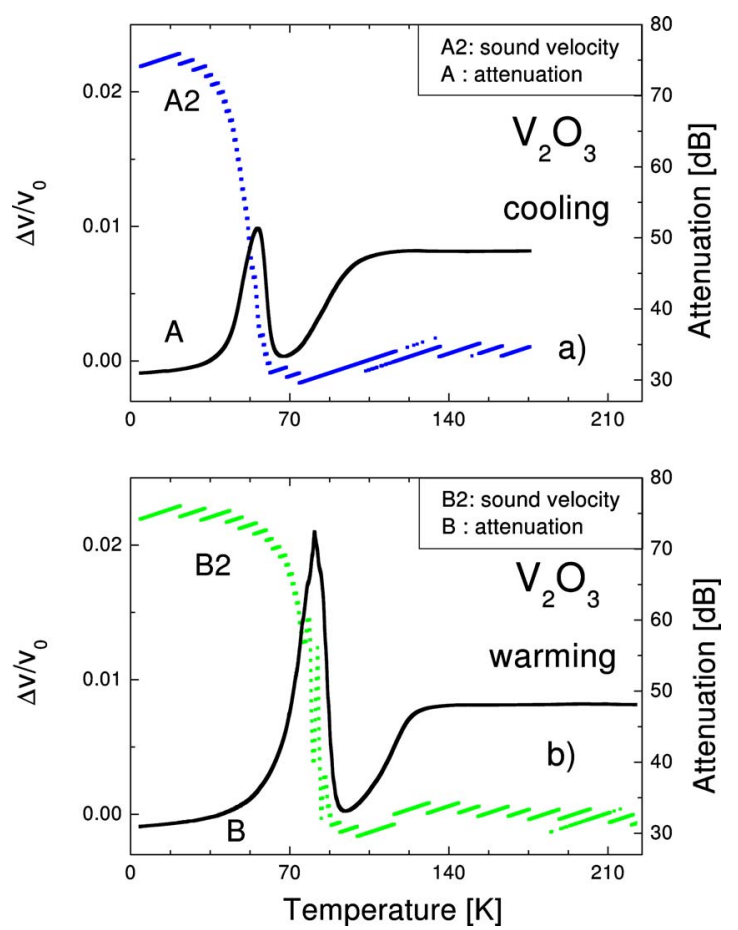

FIG. 6. (Color online) Coincidence of the minimum in the attenuation and the sound velocity shift for (a) cooling and (b) warming.

tenuation [Fig. 7(b)] goes down. At this temperature (or somewhat lower), the precursor begins to become visible in the sound velocity shift [Fig. 7(c), stripelike structures arise from the digitalization of the measured data]. However, the smooth decay of the sheet conductance does not yield the precursor observed in the sound velocity shift via a model only considering the conductivity of the film. To demonstrate this, we calculated $\Delta v / v_{0}$ according to the expression given in Sec. III C, with the sheet conductance as an input parameter, in the temperature range between 60 and $160 \mathrm{~K}$ [Fig. $7(d)]$. There is no decay of the sound velocity shift to a minimum but an (very slight) increase, which starts for decreasing temperature at about $140 \mathrm{~K}$.

The precursor observed, therefore, seems to have elastic roots. Nevertheless, the sheet conductance starts to decrease (i.e., the resistance starts to increase), together with the appearance of the precursor at around $140 \mathrm{~K}$. This increase of the resistance yields the decrease of the attenuation. It is, however, not the reason for the minimum observed in the sound velocity shift.

Thus, there seems to be an influence, i.e., a feedback of the (lattice) precursor on the $\mathrm{V}_{2} \mathrm{O}_{3}$ electronic system. In this sense, the increase of the resistance in the temperature range of the precursor is a fingerprint of changes in the lattice.

\section{E. Fingerprints of sound velocity shift oscillations in the electronic system of $\mathrm{V}_{2} \mathrm{O}_{3}$}

The existence of a feedback phenomenon between the lattice and the electronic system is most clearly visible directly at the MI transition. Here, the measured sound velocity shift $\Delta v / v_{0}$ shows a strongly oscillating behavior (most pronounced for warming) with multiple steep decays (Fig. 5). A magnified view of $\Delta v / v_{0}$ together with the theoretical curve 

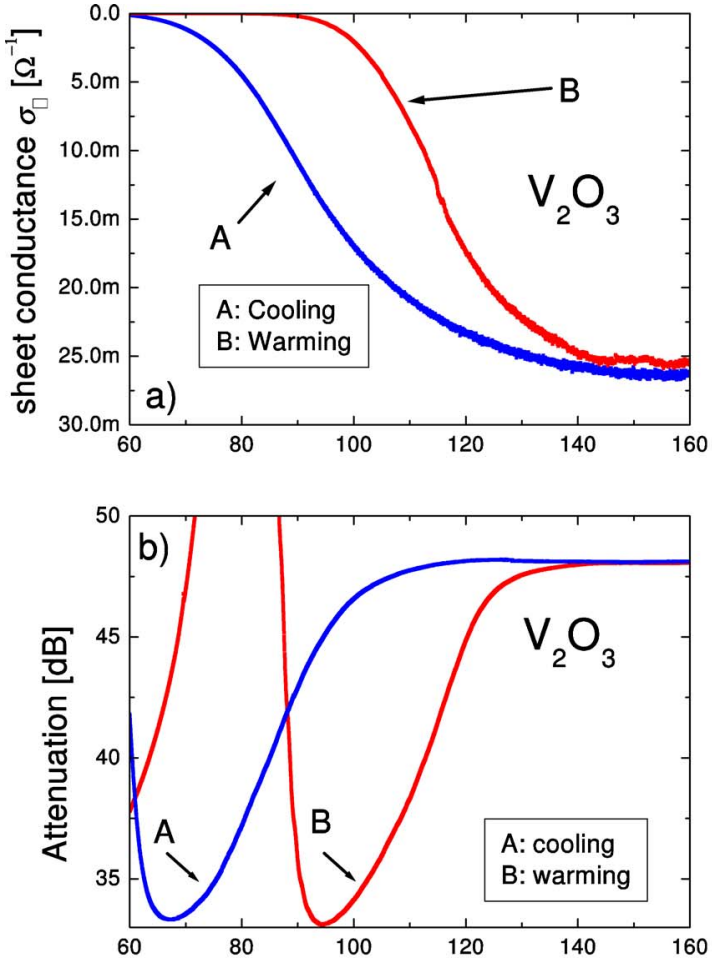

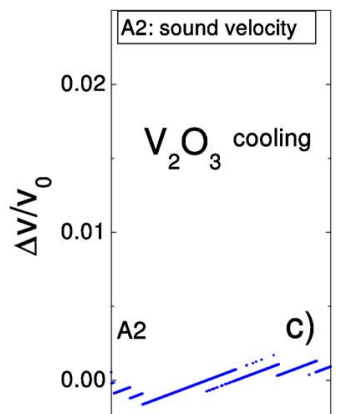

6080100120140160

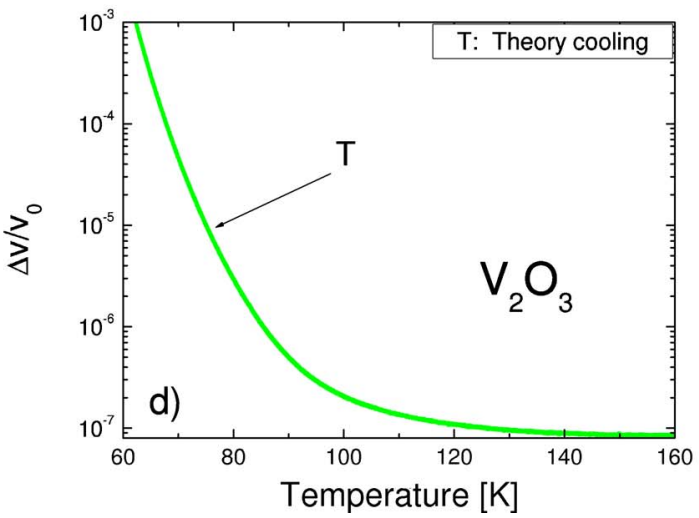

FIG. 7. Synopsis of enlarged views on different quantities in the temperature region of the precursor: (a) sheet conductance, (b) attenuation, (c) measured sound velocity shift for cooling (A2) and warming (B2), and (d) calculated sound velocity shift for cooling.

according to Sec. III C is shown in Fig. 8(a). There is a coincidence of the oscillations of the measured $\Delta v / v_{0}$ and structures in the theoretical curve. As the theory uses the sheet conductance as an input, these structures originate from fingerprints in the resistance [Fig. 8(b)]. However, similar to
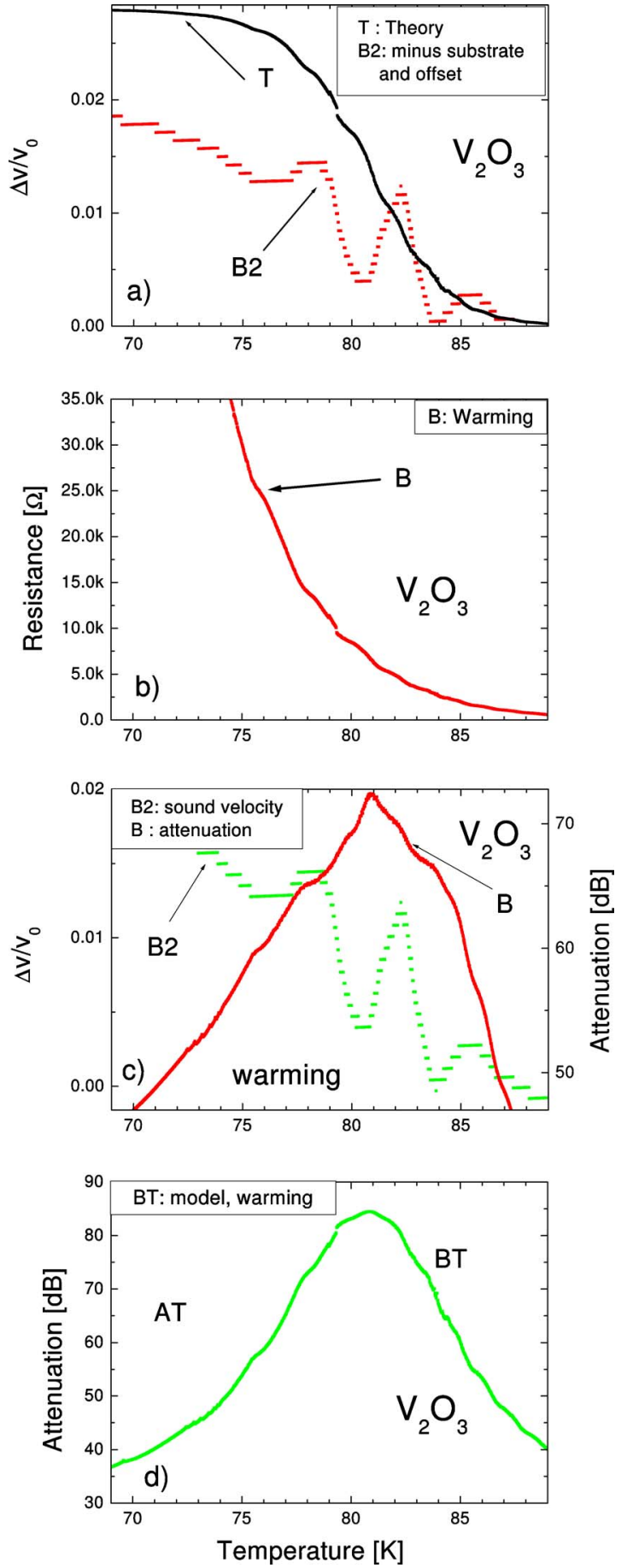

FIG. 8. Structures in different quantities at the MI transition for warming in an enlarged view: (a) measured and calculated sound velocity shift, (b) resistance (linear), (c) measured sound velocity shift and measured attenuation, and (d) calculated attenuation.

the case of the precursor region (discussed in Sec. III D), also directly at the MI transition the fingerprints in the resistance do not yield the measured oscillations in the sound velocity shift. Therefore, the oscillations probably are generated by changes in the lattice, as the observed precursor discussed above.

There are the same structures in the measured warming curve of the attenuation [Fig. 6(b)]. A magnification of the respective region is shown in Fig. 8(c). There is a coincidence of these structures with the oscillations in the mea- 
sured sound velocity shift, plotted in the same figure. The attenuation curve calculated according to Ingebrigtsen (see Sec. III B) shows similar structures as the experimental curve. Again, they enter the calculation via the measured sheet conductance calculated from the resistance.

The fingerprints in the resistivity are visible in the attenuation here because the sheet conductance is close to $\sigma_{m}$, where the curve goes through a maximum and is thus very sensitive to changes in the sheet conductance.

The reason for the oscillatory behavior of the sound velocity shift is not clear at the moment. If the film contains inhomogeneities, they could lead to a series of MI transitions in the sample. The oscillations of $\Delta v / v_{0}$ then may be experimental manifestations of the steep reduction of the sound velocity at the Mott transition, recently predicted theoretically. ${ }^{23}$ Since the Mott transition is a MI transition induced by electron-electron interaction, the sound velocity reduction appears as a reaction of the lattice to the softening of electron degrees of freedom.

\section{CONCLUSIONS}

A self-retuning SAW delay line was used to investigate the MI transition of a $\mathrm{V}_{2} \mathrm{O}_{3}$ thin film. One of the IDTs and the sound path were coated by the film. The resulting mismatch of the IDTs detunes the delay line. This mismatch vanishes during the early stages of the MI transition of the thin film and thus retunes the delay line.

Measurements of the resistance, the attenuation, and the sound velocity shift were performed in the temperature range between 260 and $4.2 \mathrm{~K}$.

Applying a model using the dc sheet conductance as an input only, the observed maxima of the attenuation could be obtained at the right temperature. The predicted height and width, however, differ from the measurements. The steplike increase measured for the sound velocity shift at the MI transition is given correctly by the model. However, there is a deviation from the calculated nearly temperature independent behavior above the transition. Instead, a slight minimum is observed experimentally.

This minimum could not be explained by the theory, which is based on a piezoelectric material covered by a thin film, of which the conductivity is considered solely in the approximation of the model applied in the present work. Therefore, as in our preceding work, ${ }^{19}$ the behavior of the sound velocity shift above the MI transition is assigned to a lattice precursor of the MI transition, caused by an interplay between orbital and lattice degrees of freedom.

While this precursor could not be observed in the attenuation in our former work, ${ }^{19}$ this was possible in the present investigation, due to the high sensitivity of the self-retuning surface acoustic wave delay line used. The reason is that changes in the lattice yield fingerprints in the electronic system. Although the resulting conductance changes are not suitable to explain the behavior of the sound velocity shift via the model discussed above, they lead to a retuning of the delay line, resulting in dramatic changes of the attenuation before the actual MI transition of the $\mathrm{V}_{2} \mathrm{O}_{3}$ thin film.

The existence of such fingerprints could be demonstrated by a magnified view on the behavior of the sound velocity shift during the steep increase of the resistivity at the actual MI transition. In this temperature range, the sound velocity shift exhibits an oscillatory behavior with steep reductions. In the measured resistivity, structures are observed, which coincide with these velocity shift oscillations. Again, the model mentioned above does not reproduce the sound velocity shift oscillations, although the conductance serves as an input.

The reason for the sound velocity shift oscillations is not clear at the moment. Possibly, the steep reduction of the sound velocity at the Mott transition, recently predicted theoretically, is observed here experimentally.

\section{ACKNOWLEDGMENTS}

The authors want to thank D. Trojak for SEM investigations, N. A. Reinke, K. Wätje, and K. Wiedenmann for Dektak profilometer measurements, R. Anders for the solution of computational problems, and W. Ruile (EPCOS) for the split four $\mathrm{LiNbO}_{3}$ device. The present work was supported by the SFB 484 .

${ }^{1}$ O. Müller, J. P. Urbach, E. Goering, T. Weber, R. Barth, H. Schuler, M. Klemm, S. Horn, and M. L. denBoer, Phys. Rev. B 56, 15056 (1997).

${ }^{2}$ J.-H. Park, L. H. Tjeng, A. Tanaka, J. W. Allen, C. T. Chen, P. Metcalf, J. M. Honig, F. M. F. de Groot, and G. A. Sawatzky, Phys. Rev. B 61, 11506 (2000).

${ }^{3}$ F. Mila, R. Shiina, F.-C. Zhang, A. Joshi, M. Ma, V. Anisimov, and T. M. Rice, Phys. Rev. Lett. 85, 1714 (2000).

${ }^{4}$ R. J. Radwanski and Z. Ropka, e-print arXiv:cond-mat/0012257.

${ }^{5}$ R. Shiina, F. Mila, F.-C. Zhang, and T. M. Rice, Phys. Rev. B 63, 144422 (2001).

${ }^{6}$ E. Goering, M. Schramme, O. Müller, R. Barth, H. Paulin, M. Klemm, M. L. den Boer, and S. Horn, Phys. Rev. B 55, 4225 (1997).

${ }^{7}$ A. Tanaka, J. Phys. Soc. Jpn. 71, 1091 (2002).

${ }^{8}$ A. Tanaka, Physica B 329-333, 753 (2003).

${ }^{9}$ S. Klimm, M. Herz, R. Horny, G. Obermeier, M. Klemm, and S. Horn, Phys. Rev. B 64, 184435 (2001).

${ }^{10}$ M. Foëx, Comptes Rendus Hebdomadaires des Séances de l'Académie des Sciences 223, 1126 (1946).

${ }^{11}$ J. F. Morin, Phys. Rev. Lett. 3, 34 (1959).

${ }^{12}$ W. Brückner, H. Oppermann, W. Reichelt, J. I. Terukow, F. A. Tschudnowski, and E. Wolf, Vanadiumoxide (Akademie, Berlin, 1983).

${ }^{13}$ N. F. Mott, Rev. Mod. Phys. 40, 677 (1968).

${ }^{14}$ D. B. McWhan, T. M. Rice, and J. P. Remeika, Phys. Rev. Lett. 23, 1384 (1969).

${ }^{15}$ M. Imada, A. Fujimori, and Y. Tokura, Rev. Mod. Phys. 70, 1039 (2003).

${ }^{16}$ K. Held, G. Keller, V. Eyert, D. Vollhardt, and V. I. Anisimov, Phys. Rev. Lett. 86, 5345 (2001).

${ }^{17}$ D. B. McWhan, A. Menth, J. P. Remeika, W. F. Brinkmann, and T. M. Rice, Phys. Rev. B 7, 1920 (1973).

${ }^{18}$ A. I. Frenkel, E. A. Stern, and F. A. Chudnovsky, Solid State Commun. 102, 637 (1997).

${ }^{19}$ C. Müller, A. A. Nateprov, G. Obermeier, M. Klemm, R. Tidecks, A. Wixforth, and S. Horn, J. Appl. Phys. 98, 084111 (2005). In this work, a factor of -1 has to be added in the left hand side of Eq. (6). Moreover, in the second line above Eq. (4), instead of " $\Delta v=v-v_{0}$," it should read " $\Delta v=v-v_{\text {sc }}$ " [where $v_{\mathrm{sc}}$ is the short circuited sound velocity introduced in Eq. (5)]. Finally, in Chap. II (third paragraph), it should read "with $2 \Theta$ ranging from."

${ }^{20} \mathrm{P}$. Pfalzer, "Lokale strukturelle und elektronische Eigenschaften von $\mathrm{V}_{2} \mathrm{O}_{3}$ und $\mathrm{ZnV}_{2} \mathrm{O}_{4}$," Ph.D. thesis, Universität Augsburg, 2004.

${ }^{21}$ P. Pfalzer, G. Obermeier, M. Klemm, S. Horn, and M. L. den Boer, Phys. Rev. B 73, 144106 (2006).

${ }^{22}$ Md. Motin Seikh, C. Narayana, A. K. Sood, P. Murugavel, M. W. Kim, P. A. Metcalf, J. M. Honig, and C. N. R. Rao, Solid State Commun. 138, 466 (2006).

${ }^{23}$ S. Hassan, A. Georges, and H. R. Krishnamurthy, Phys. Rev. Lett. 94, 
$036402(2005)$

${ }^{24}$ N. Kuroda and H. Y. Fan, Phys. Rev. B 16, 5003 (1977).

${ }^{25}$ R. M. White and F. W. Voltmer, Appl. Phys. Lett. 7, 314 (1965).

${ }^{26}$ G. Kovacs, M. Anhorn, H. E. Engan, G. Visintini, and C. C. W. Ruppel, Ultrasonics Symposium Proceedings (The Institute of Electrical and Electronics Engineers, New York, 1990), pp. 435-438.

${ }^{27}$ S. Datta, Surface Acoustic wave Devices (Prentice-Hall, Englewood Cliffs, NJ, 1986).

${ }^{28}$ K. Dransfeld and E. Salzman, in Excitation, Detection and Attenuation of High-Frequency Elastic Surface Waves, Physical Acoustics, Principles and Methods Vol. VII, edited by W. P. Mason and R. N. Thurston (Academic, New York, 1970), Chap. 4.

${ }^{29}$ S. Klimm, "Magnetotransportmessungen zur Untersuchung der elektronis- chen Struktur von $\mathrm{V}_{2} \mathrm{O}_{3}$ und $\mathrm{MoO}_{2}$, Ph.D. thesis, Universität Augsburg, 1997.

${ }^{30} \mathrm{C}$. Müller, V. Zestrea, V. Tsurkan, S. Horn, R. Tidecks, and A. Wixforth, J. Appl. Phys. 99, 023906 (2006).

${ }^{31}$ K. A. Ingebrigtsen, J. Appl. Phys. 41, 454 (1970).

${ }^{32}$ A. Wixforth, J. Scriba, M. Wassermeier, J. P. Kotthaus, G. Weimann, and W. Schlapp, Phys. Rev. B 40, 7874 (1989).

${ }^{33}$ A. Hörner, personal communication (2005).

${ }^{34}$ K. A. Ingebrigtsen, J. Appl. Phys. 40, 2681 (1969).

${ }^{35}$ A. R. Hutson and D. L. White, J. Appl. Phys. 33, 40 (1962).

${ }^{36}$ Surface Wave Filters, edited by H. Matthews (Wiley, New York, 1977).

${ }^{37}$ Surface Acoustic Waves, edited by A. A. Oliner (Springer-Verlag, Berlin, 1973). 